\title{
Carnosol as a Nrf2 Activator Improves Endothelial Barrier Function Through Antioxidative Mechanisms
}

\author{
Xi Li ${ }^{1,2}$, Qiao Zhang ${ }^{1,2}$, Ning Hou ${ }^{1,2}$, Jing Li ${ }^{1,2}$, Min Liu ${ }^{1,2}$, Sha Peng ${ }^{1,2}$, Yuxin Zhang 1,2, \\ Yinzhen Luo ${ }^{1,2}$, Bowen Zhao ${ }^{1,2}$, Shifeng Wang ${ }^{1,2}$, Yanling Zhang ${ }^{1,2, *}$ and Yanjiang Qiao ${ }^{1,2, *}$ \\ 1 School of Chinese Pharmacy, Beijing University of Chinese Medicine, Beijing 100102, China; \\ xixili1994@163.com (X.L.); zhangqiao0824@foxmail.com (Q.Z.); 13821117290@163.com (N.H.); \\ lijing20171008@163.com (J.L.); liumin_94@163.com (M.L.); moonshadow0406@163.com (S.P.); \\ zhangyuxinwjzy@163.com (Y.Z.); lyz985472@163.com (Y.L.); zhaobw9504@163.com (B.Z.); \\ alana6268@126.com (S.W.) \\ 2 Beijing Key Laboratory of Chinese Materia Medica Foundation and New Drug Research and Development, \\ Beijing 100102, China \\ * Correspondence: zhangyanling@bucm.edu.cn (Y.Z.); yjqiao@bucm.edu.cn (Y.Q.); \\ Tel.: +86-10-8473-8620 (Y.Z. \& Y.Q.)
}

Received: 24 December 2018; Accepted: 14 February 2019; Published: 18 February 2019

\begin{abstract}
Oxidative stress is the main pathogenesis of diabetic microangiopathy, which can cause microvascular endothelial cell damage and destroy vascular barrier. In this study, it is found that carnosol protects human microvascular endothelial cells (HMVEC) through antioxidative mechanisms. First, we measured the antioxidant activity of carnosol. We showed that carnosol pretreatment suppressed tert-butyl hydroperoxide ( $\mathrm{t}-\mathrm{BHP}$ )-induced cell viability, affected the production of lactate dehydrogenase (LDH) as well as reactive oxygen species (ROS), and increased the produce of nitric oxide (NO). Additionally, carnosol promotes the protein expression of vascular endothelial cadherin (VE-cadherin) to keep the integrity of intercellular junctions, which indicated that it protected microvascular barrier in oxidative stress. Meanwhile, we investigated that carnosol can interrupt Nrf2-Keap1 protein - protein interaction and stimulated antioxidant-responsive element (ARE)-driven luciferase activity in vitro. Mechanistically, we showed that carnosol promotes the expression of heme oxygenase $1(\mathrm{HO}-1)$ and nuclear factor-erythroid 2 related factor 2(Nrf2). It can also promote the expression of endothelial nitric oxide synthase (eNOS). Collectively, our data support the notion that carnosol is a protective agent in HMVECs and has the potential for therapeutic use in the treatments of microvascular endothelial cell injury.
\end{abstract}

Keywords: carnosol; antioxidant activity; microvascular endothelial protection; HMVEC cells

\section{Introduction}

Diabetic microangiopathy causes endothelial dysfunction and vascular complications, which is one of the main causes of death and disability in diabetes mellitus [1]. Therefore, it is important for us to study the influence of endothelial dysfunction on diabetic microangiopathy.

Endothelial cell-to-cell junctions have crucial roles in the process of hyperglycemia vascular endothelial injury because they not only maintain intercellular adhesion, but also transfer intracellular signals that modulate contact inhibition of cell growth, cell polarity, lumen formation, and interactions with pericytes and smooth muscle cells. VE-cadherin is a basic organization of adherens junction protein, which promotes vascular stability, such as protecting cells from apoptosis and controlling the permeability of endothelium [2,3]. Notably, LDH is a stable cytoplasmic enzyme. The leakage of intracellular LDH into the surrounding medium is thought to be the result of cell membrane 
damage or lysis [4], and this is an irreversible process. Therefore, the increase of LDH can reflect the degree of damage of the cell membrane. In particular, NO can help regulate the normal function of the endothelial barrier and the vascular function eNOS promotes the product of NO in vascular endothelium and blood vessels [5].

In 2001, Brownlee [6] proposed the "Common Mechanism of Diabetic Complications" theory that suggests overproduction of superoxide and ROS causes hyperglycemia vascular endothelial injury, which plays a key role in in the mechanisms of diabetic complications. Oxidative stress can significantly increase ROS levels $[7,8]$. The accumulation of ROS can cause many diseases, including cardiovascular disease, endothelial dysfunction [9] and aging-related diseases [10], as well as neurodegenerative diseases [11]. As a key pathway for oxidative stress, the Keap1/Nrf2/ARE system plays an important role in the study of diabetes [12]. The activation of Nrf2 of human microvascular endothelial cells can significantly improve endothelial dysfunction [13]. When Nrf2 uncouples from Keap1, Keap1 releases Nrf2 and Nrf2 enters the nucleus. Nrf2 can bind its cognate response element, the antioxidant response element (ARE), which upregulates the transcription of ARE-responsive genes, such as HO-1, glutathione (GSH), glutamate-cystein ligase catalytic (GCLC), glutathione-S-transferase (GST) and NADP $(\mathrm{H})$ quinone oxidoreductase 1 (NQO1) [14,15].

Natural products play an important role in drug discovery [16]. Rosemary (Rosmarinus officinalis L., Lamiaceae) is a woody perennial herb, commonly used for flavoring foods as a condiment [17]. Carnosol (Figure 1) is an anti-inflammatory and anti-oxidant compound which is one of the main components of the extract of rosemary. It has been reported that carnosol also possess potent anti-microbial neuroprotective and anti-tumor properties [18-20]. The aim of our study was to investigate the protective effect of carnosol on endothelial damage in HMVEC cells.<smiles>CC(C)c1cc2c(c(O)c1O)C13CCCC(C)(C)C1CC(O2)O3</smiles>

Figure 1. Chemical structure of carnosol.

In present research, we first found that carnosol can protect against t-BHP-mediated microvascular endothelial injury in HMVEC cells. Moreover, we evaluated that carnosol can firstly interrupt Nrf2-Keap1 protein-protein interaction. We found that carnosol significantly induces the antioxidant genes and vascular endothelium protection genes upregulation in vitro, such as HO-1, Nrf2 and eNOS. For the first time, carnosol was demonstrated that stimulates the VE-cadherin expression in cell-to-cell junctions. We have set up a complete evaluation system which can prove the protective effect of carnosol on microvascular endothelial barrier from phenotype to mechanism in vitro. It has been shown that Tertiary butylhydroquinone (TBHQ) is a positive agonist of Keap1/Nrf2/ARE system and has an effect of improving diabetes mellitus [21]. Therefore, we chose TBHQ as a positive control.

\section{Results}

\subsection{Carnosol Has the Antioxidant Activity in ABTS Assay}

2,2'-azino-bis(3-ethylbenzothiazoline-6-sulphonic acid) (ABTS) produces green ABTS ${ }^{+}$under the appropriate antioxidants, and $\mathrm{ABTS}^{+}$is inhibited with the antioxidants. The total antioxidant capacity of the samples can be determined and calculated by measuring the absorbance of $\mathrm{ABTS}^{+}$at $734 \mathrm{~nm}$. The capacity of carnosol to scavenge ABTS ${ }^{+}$was shown in Figure 2, which suggested that carnosol has antioxidant activity. But the antioxidant activity of carnosol was lower than that of $10 \mu \mathrm{M} \mathrm{TBHQ}$. 


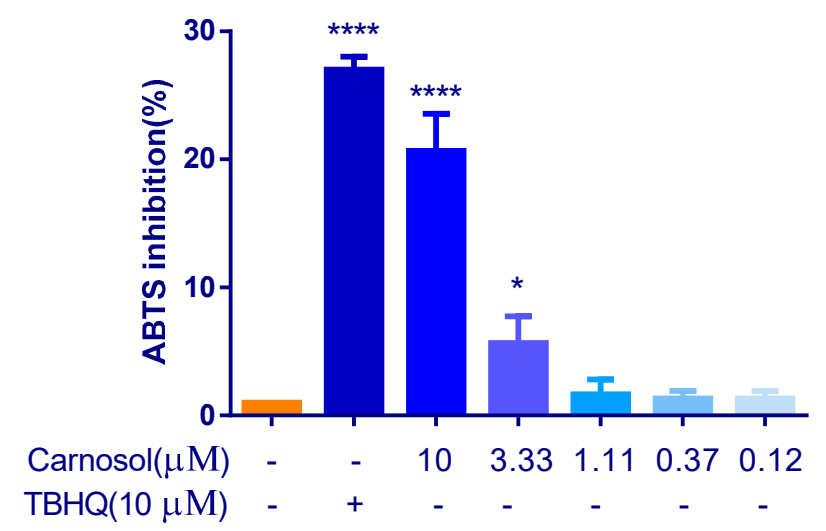

Figure 2. Detection of antioxidant activity of carnosol by ABTS radical scavenging activity. ${ }^{*} p<0.05$, $* * * * p<0.0001,0.25 \%$ DMSO-treated as negative control group, TBHQ $(10 \mu \mathrm{M})$ was used as a positive control group, compared with negative group. Results are expressed as mean $\pm \operatorname{SD}(n=3)$.

\subsection{Carnosol Can Protect HMVEC Cells Against t-BHP Induced Cell Injury}

In order to study the effects of carnosol in HMVEC cells, the cytotoxicity of carnosol was first assessed by the CCK-8 assay. The result showed that cells were no cytotoxicity at $10 \mu \mathrm{M}$ of carnosol (Figure 3a). After treating with $200 \mu \mathrm{M} \mathrm{t}-\mathrm{BHP}$ for $3 \mathrm{~h}$, cells have a $20 \%$ mortality rate ((Figure $3 \mathrm{~b}$ ). Pretreatment of cells with $10 \mu \mathrm{M}$ carnosol considerably reduced t-BHP-induced cell injury (Figure $3 \mathrm{~b}$ ). Then, we drew the supernatant to detect LDH. The result suggests that carnosol can significantly reduce the release of LDH (Figure 3c). To evaluate the effect of apoptosis, we used two methods to evaluate apoptosis. After pretreating cells with $10 \mu \mathrm{M}$ carnosol for $24 \mathrm{~h}$ and $200 \mu \mathrm{M}$ t-BHP for an additional $3 \mathrm{~h}$, we treated with the Annexin V-FITC and PI for $15 \mathrm{~min}$. Then we observed the cell apoptosis of HMVEC cells using fluorescence microscopy. We can obviously observe that $10 \mu \mathrm{M}$ carnosol can improve cell apoptosis (Figure 3d). Next, we used flow cytometry for a quantitative detection. We found $10 \mu \mathrm{M}$ carnosol can improve cells apoptosis significantly compared with t-BHP -treated group (Figure 3e,f). 

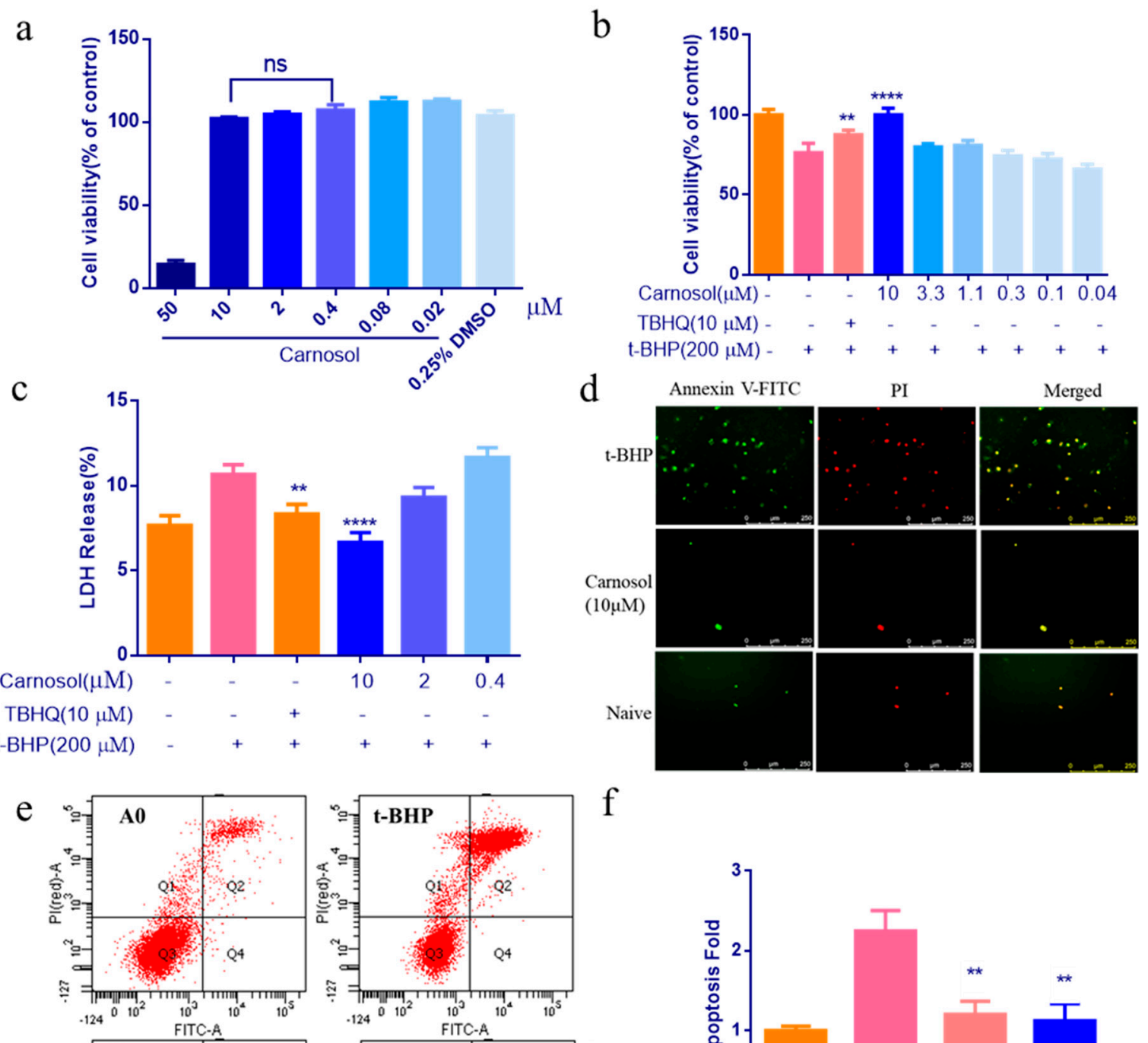

f
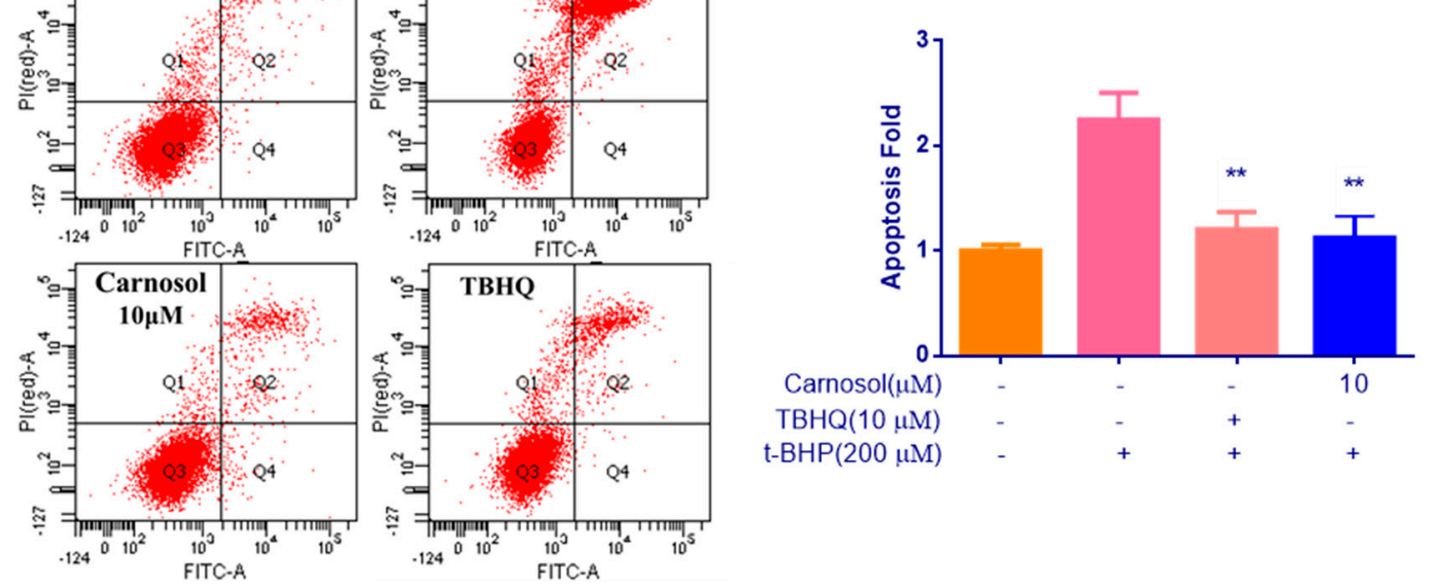

Figure 3. To evaluate the protective effect of carnosol in t-BHP-induced endothelial injury model. (a) Evaluating the cell viability of carnosol by CCK-8 assay. (b) The cell viability of carnosol pretreated cells after t-BHP-treated for $3 \mathrm{~h}$. (c) The levels of the release of LDH were measured using LDH kits. (d) the green fluorescence is Annexin V-FITC staining positive cell, and the red fluorescence is propidium iodide $(\mathrm{PI})$ staining positive cell at lower magnification $(10 \times)$. Apoptotic cells were stained only by green fluorescence, necrotic cells were stained with green and red fluorescence, and normal cells were not stained with fluorescence. $(\mathbf{e}, \mathbf{f})$ Detection of apoptosis by flow cytometry. Apoptotic cells were distributed in Q2 and Q4 regions. ${ }^{* *} p<0.01,{ }^{* * * *} p<0.0001$, ns: no significant difference. $200 \mu \mathrm{M}$ t-BHP-treated as model group, TBHQ $(10 \mu \mathrm{M})$ was used as a positive control group, $0.25 \%$ DMSO-treated as negative control group, compared with model group. Results are expressed as mean $\pm \mathrm{SD}(n=3)$. 


\subsection{Carnosol Increases the Expression of VE-Cadherin in HMVEC Cells}

To address the role of carnosol in regulating endothelial barrier function, we first studied the VE-cadherin localization in HMVEC cells. We thus used immunofluorescence to detect the expression and localization of VE-cadherin. After being stimulated with t-BHP $3 \mathrm{~h}$, it disrupted VE-cadherin distribution. In addition, $10 \mu \mathrm{M}$ carnosol could significantly increase the expression of VE-cadherin. It also maintains the endothelial contacts and adhesion between neighboring cells (Figure 4). The analyzed pictures are the quantification of the expression of VE-cadherin. We measured the fluorescence intensity on a line (white in Figure 4) through the nucleus of the cell in the merge picture. The green line is the fluorescence intensity of VE-cadherin, the blue line is the fluorescence intensity of nucleus, and the peak height indicates the fluorescence intensity. We found that the VE-cadherin intensity of the t-BHP group was reduced. The expression of VE-cadherin was higher in the carnosol group and naive group than in the t-BHP group, and it was shown as a high peak at the junction of cell membrane.

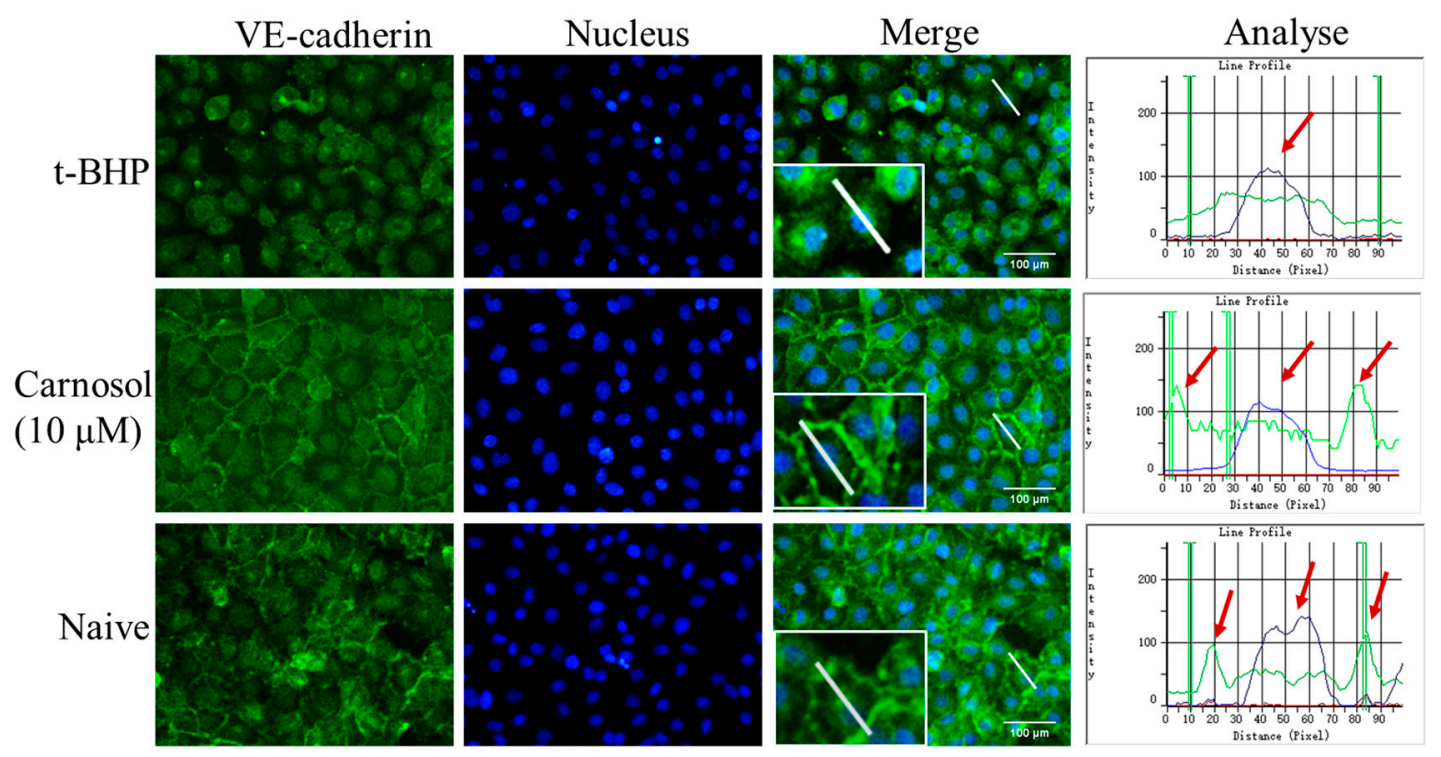

Figure 4. Immunofluorescence staining of VE-cadherin at $20 \times$ magnification. VE-cadherin shows a green line on the cell membranes. We continued to enlarge twice in the lower right corner of the merge images. The last row graphs show the relative intensity profiles of fluorescent signal intensities of VE-cadherin (green) and nucleu (blue) along the white line scans depicted in the immunofluorescent images. The $X$-axis indicates the distance of the line and the $Y$-axis represents the fluorescence intensity. The red arrows indicate the location of the peak.

\subsection{Carnosol Reduces the ROS Production and Promotes NO Release in t-BHP-Induced Cell Injury in HMVEC Cells}

To confirm the protective properties of carnosol, we investigated whether carnosol countered intracellular ROS and NO production in HMVEC cells treated with $200 \mu \mathrm{M}$ t-BHP for an additional 3 h. We found ROS production decreased significantly compared with t-BHP -treated group (Figure 5a). Therefore, the results suggested that carnosol might have cytoprotective effects by inhibiting the accumulation of ROS. As shown in Figure 5b, the green fluorescence represents the NO bound by the probe. We can observe that carnosol can significantly promote the release of NO (Figure 5c). 
a

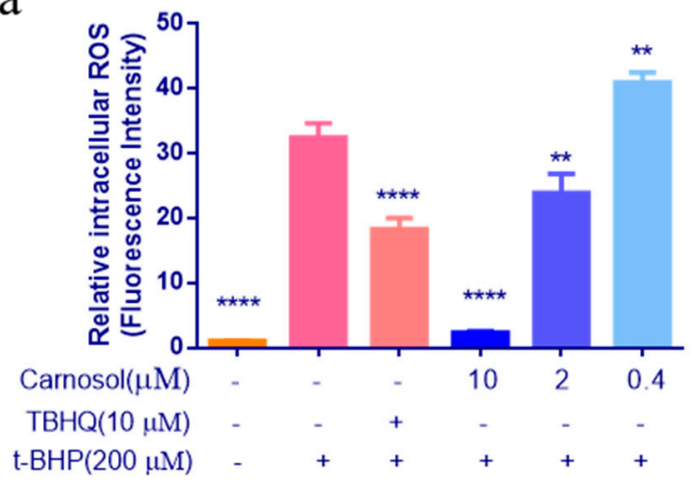

$\mathrm{C}$

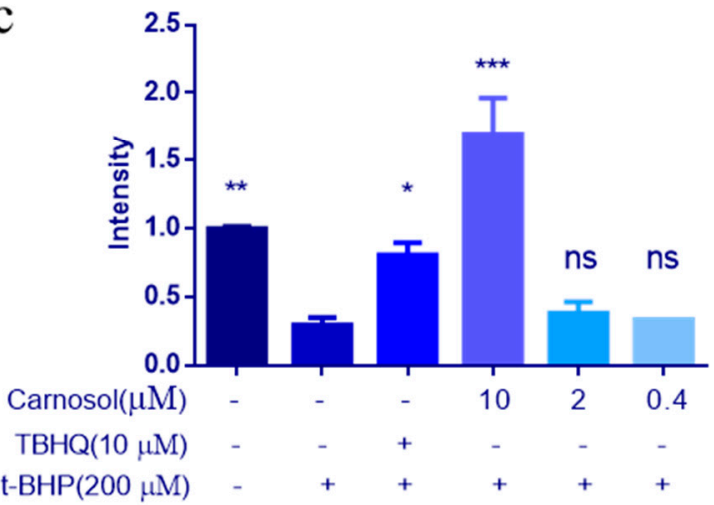

b
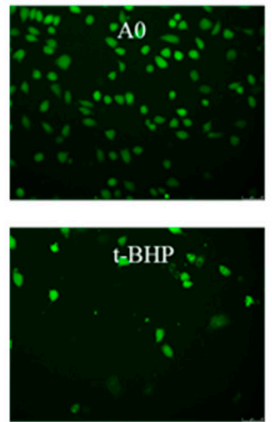

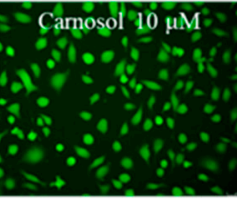

- Cảmosol $2 \mu \mathrm{M}$

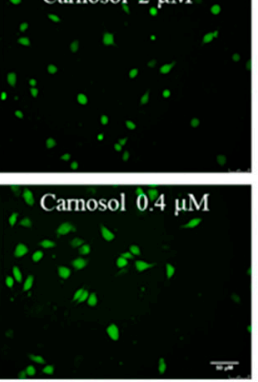

Figure 5. Effects of carnosol in ROS and NO assays. (a) The levels of intracellular ROS were measured using DCFH-DA fluorescent probe. $(\mathbf{b}-\mathbf{c})$ The levels of intracellular NO were measured using DAF-FM DA fluorescent probe. It was used a fluorescence microscope to take photos at $20 \times$ magnification. ${ }^{*} p<0.05,{ }^{* *} p<0.01,{ }^{* * *} p<0.001,{ }^{* * * *} p<0.0001$, ns: no significant difference. $200 \mu \mathrm{M}$ t-BHP-treated as model group, TBHQ $(10 \mu \mathrm{M})$ was used as a positive control group, $0.25 \%$ DMSO-treated as a negative control group, all groups were compared with the model group (no carnosol, no TBHQ, with $200 \mu \mathrm{M}$ t-BHP).

2.5. Carnosol Has the Potential of Interrupting Nrf2-Keap1 Protein-Protein Interaction and Stimulating ARE-Mediated Luciferase Activity

We used molecular docking techniques to analyze whether carnosol can directly inhibit the Nrf2-Keap1 PPI. We found carnosol formed hydrogen bond interactions with amino acids ARG415, SER602, GLY603 and hydrophobic interaction with TRY334, TRY525, TRY572, ALA556(Figure 6a,b). In particular, the interaction pattern was similar to the original ligand, indicating that carnosol has a high potential inhibitory activity. In luciferase reporter activity assay, the dose-response of t-BHP indicates that our cell lines have been successfully constructed (Figure $6 c$ ). Then, we proved that carnosol significantly increased ARE luciferase activity in a dose-dependent manner (Figure 6d). 
a

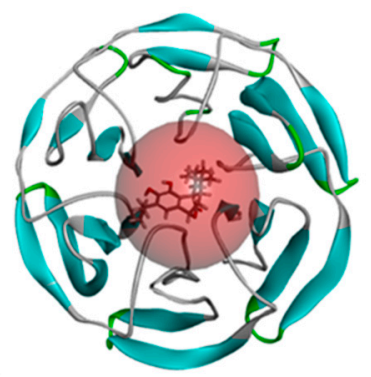

$\mathrm{C}$

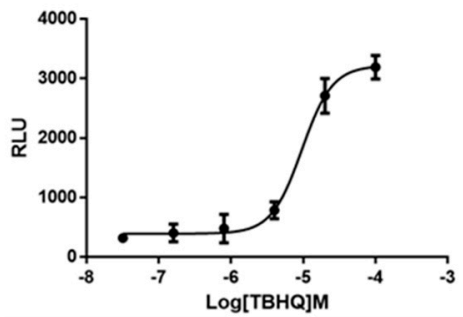

b

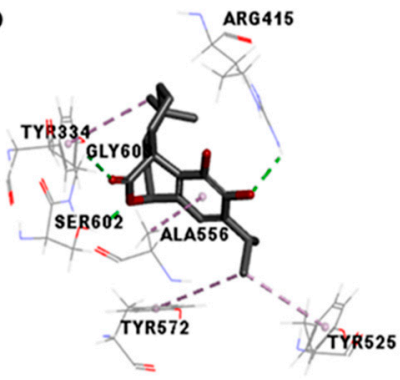

d

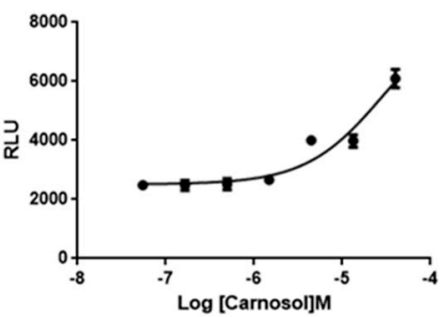

Figure 6. Docking mode of carnosol in the binding site of Keap1 and ARE-mediated luciferase activity. (a) Top view of the docking mode of carnosol in the binding site of Keap1. (b) The potential interaction between carnosol and Keap1 is via hydrogen bonds and hydrophobic. Potential hydrogen bonds were depicted in green dot lines and hydrophobic were depicted in purple dot lines. (c) Dose-response curves of ARE agonist (t-BHP) served as a positive control in this study. (d) Carnosol induced ARE-luciferase activity in a dose-dependent manner. Results are expressed as mean $\pm \operatorname{SD}(n=3)$.

\subsection{Carnosol Stimulates the Expression of Cytoprotective Genes and Proteins.}

RT-PCR analysis of carnosol-treated cells showed a significant elevation of eNOS, HO-1 and Nrf2 genes in HMVEC cells. The mRNA levels of increased respectively compared with the control (no carnosol, no TBHQ, with $200 \mu \mathrm{M}$ t-BHP). These results suggest that $10 \mu \mathrm{M}$ carnosol treatment could up-regulate Nrf2, HO-1 and eNOS genes expression in HMVEC cells (Figure 7a-c). We have also detected the protein levels of HO-1 and Nrf2 in response to the carnosol treatment for $24 \mathrm{~h}$ under the doses (Figure 7d). HO-1 and Nrf2-mediated cytoprotective proteins were activated by carnosol at $10 \mu \mathrm{M}$. Next, the time dependent inductions of HO-1 and Nrf2 by carnosol was investigated. The protein level of HO- 1 in HMVEC cells treated by carnosol increased as early as $4 \mathrm{~h}$, reached the highest level at $8 \mathrm{~h}$, and then gradually decreased (Figure 7e). The protein level of Nrf2 in HMVEC cells treated by carnosol increased as early as $2 \mathrm{~h}$, reached the highest level at $8 \mathrm{~h}$, and then gradually decreased (Figure 7f). 


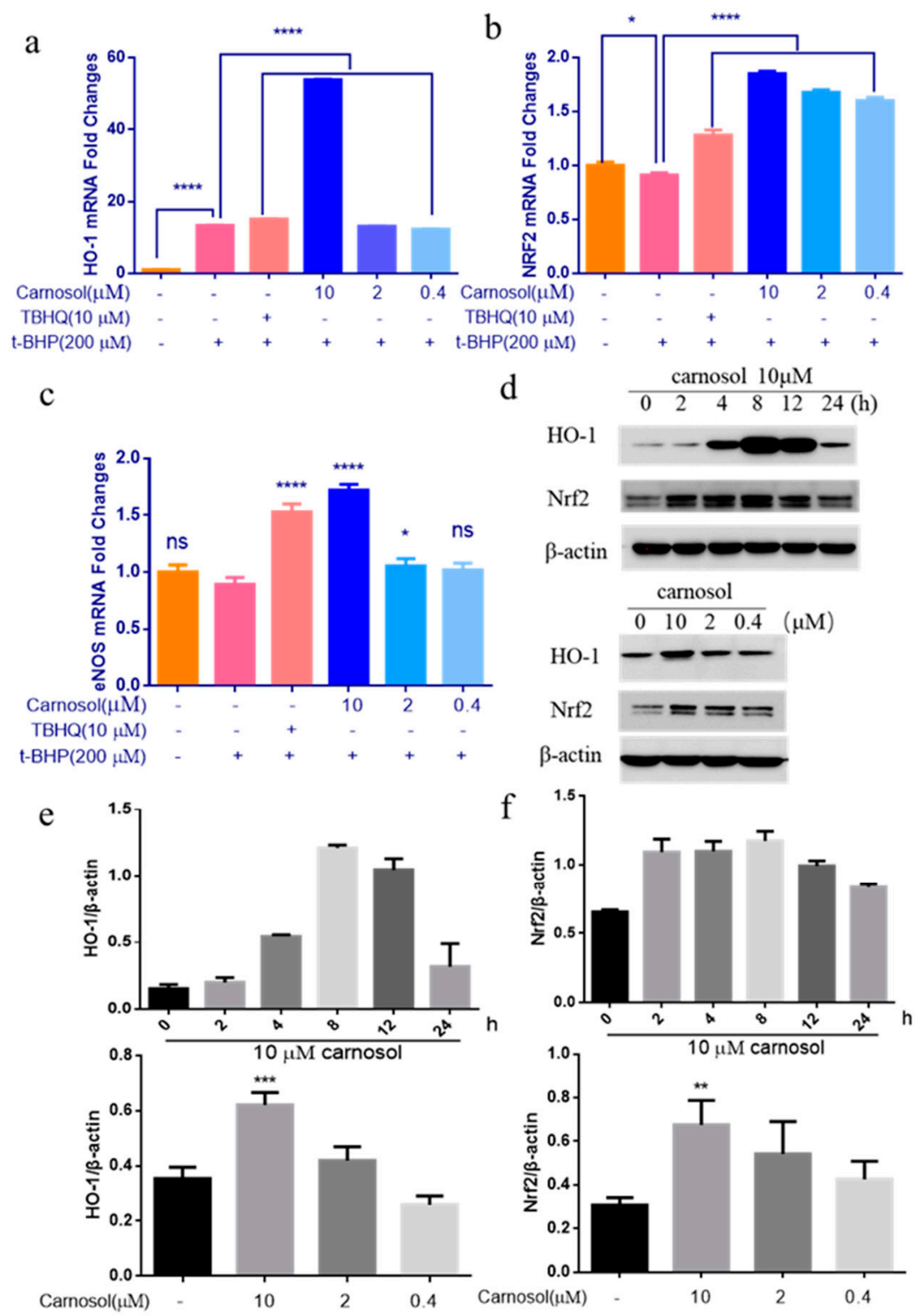

Figure 7. Effects of carnosol on cytoprotective gene and protein expression in HMVEC cells. (a-c) The mRNA levels of cytoprotective genes, such as HO-1, Nrf2 and eNOS were evaluated using real-time RT-PCR, with GAPDH as an internal control. (d) HMVEC cells were treated with $10 \mu \mathrm{M}$ carnosol for 0, $2,4,8,12$ and $24 \mathrm{~h}$ (top) or with various concentrations of t-CA for $24 \mathrm{~h}$ (bottom). The protein levels of HO-1 and Nrf2 were detected with immunoblotting. (e) Levels of HO-1 expression with $10 \mu \mathrm{M}$ carnosol for $0,2,4,8,12$ and $24 \mathrm{~h}$ (top). Levels of HO-1 expression with various concentrations of t-CA for $24 \mathrm{~h}$ (bottom). (f) Levels of Nrf2 expression with $10 \mu \mathrm{M}$ carnosol for 0, 2, 4, 8, 12 and $24 \mathrm{~h}$ (top). Levels of Nrf2 expression with various concentrations of t-CA for $24 \mathrm{~h}$ (bottom). ${ }^{*} p<0.05,{ }^{* *} p<0.01$, ${ }^{* * *} p<0.001,{ }^{* * * *} p<0.0001,200 \mu \mathrm{M}$ t-BHP-treated as model group, TBHQ $(10 \mu \mathrm{M})$ was used as a positive control group, $0.25 \%$ DMSO-treated as a negative control group, compared with the model group. Results are expressed as mean $\pm \mathrm{SD}(n=3)$.

\section{Discussion}

Many natural compounds and natural product mimics are potential antioxidants which can protect against oxidative damage in chronic diseases [22]. Phenolic acids are an important component of natural antioxidants. Carnosol is a kind of diterpenoid, which was known to possess a range of therapeutic effects such as anti-cancer, anti-inflammatory, and anti-oxidant activities in previous studies [23]. Diabetic microangiopathy includes diabetic retinopathy, diabetic nephropathy, 
and diabetic neuropathy. Most of these diseases interact with endothelial injury and oxidative stress [24-26]. Here, we showed that carnosol improves t-BHP-induced endothelial injury in HMVEC Cells.

It has been shown that t-BHP can cause endothelial cell injury through different pathways [27,28]. To establish the model for endothelial injury, we used t-BHP to induce cell injury in this study. LDH is a key marker of cell membrane integrity and cell viability. It is a cytoplasm containing enzymes of living cells. The increase of LDH is usually regarded as a marker of cell disintegration and necrosis $[29,30]$. We proved that carnosol pretreatment increased cell viability and reduced LDH release caused by $\mathrm{t}$-BHP in HMVEC cells. Abnormal apoptosis may lead to pathological conditions [31]. Apoptosis is an important factor in endothelial protection, and the development of it is directly related to the degree of endothelial damage. We found that carnosol can improve cells apoptosis significantly compared with t-BHP -treated group of seriously endothelial damage. Therefore, we believe that carnosol is a potential drug for endothelial protection.

Disrupting the endothelial cell junctions not only increases vascular permeability, but also changes the response of endothelial cells to their environment and surrounding cells. For instance, VE-cadherin is an important component for the vascular endothelial adhesion and protects the endothelial barrier [3]. Our results show that carnosol can significantly protect the structure of cells by promoting the expression of VE-cadherin. Additionally, the function and organization of VE-cadherin can be regulated by numerous vasoactive agents such as histamine, prostaglandins, thrombin and NO [32]. eNOS produces NO in the blood vessels, while NO helps play crucial roles in regulating vascular function, endothelium inflammatory reaction, as well as vascular growth and regeneration [5]. Carnosol can significantly promote the release of $\mathrm{NO}$ and promote the expression of eNOS in HMVEC cells. This may be an important mechanism for enabling carnosol to protect the endothelium barrier.

Oxidative stress is a main cause of the development of diabetic microangiopathy. However, the clinical strategies for the treatment of diabetic microangiopathy by enhancing antioxidant defense mechanisms have not been fully explored. Overproduction ROS production that exceeds the antioxidant capacity leads to cellular oxidative stress and is implicated in a broad range of diseases, such as endothelial injury and cancer. [33]. In particular, ROS contribute to the promotion of the oxidative stress response and the activation Nrf2 signaling [34]. We have demonstrated that carnosol can significantly reduce the production of ROS in HMVEC cells. Keap1/Nrf2/ARE pathway plays an important role in the mediation of health recovery, oxidative stress, diabetes and its complications $[35,36]$. Nrf2-Keap1 protein-protein interaction (PPI) has been recognized as a key point for regulating Nrf2 activation [37]. Keap1 depends on the interaction with the key tyrosines, namely Tyr525, Tyr574, Arg-415 and Tyr334 [38,39]. We confirmed that Arg415, Tyr334, Tyr525 were key amino acids for the interaction of carnosol with Keap1. Moreover, carnosol can activate the HEK293-ARE cell line with a gradient. Therefore, we considered that carnosol has the potential to activate the Nrf2 and interrupt Nrf2-Keap1 protein-protein interaction. The virtual method also provided a promising strategy to discover Nrf2 inducers. But we still need to prove the effect of carnosol on keap1 protein in our future experiments. Nowadays, Nrf2 is called the "main regulator" of antioxidant reaction. It stimulates the expression of hundreds of genes, most of which encode antioxidant/detoxifying enzymes, such as HO-1 [40]. Indeed, HO-1 is an important enzyme against oxidative damage [41]. So, we focused on the capability of carnosol on up-regulating HO-1 and Nrf2 gene and protein expression in HMVEC cells.

In summary, this study revealed that carnosol strongly induced Nrf2 activation in HMVEC cells and prevented endothelial injury. Carnosol protects HMVEC cells by promoting NO production and reducing the ROS and LDH released. Carnosol can upregulate eNOS gene expression and VE-cadherin protein expression. This occurs via ROS-mediating the activation of Nrf2-ARE pathway that appears to be responsible for inducing Nrf2 accumulation in the nucleus. It subsequently binds to ARE sequences and upregulates HO-1 and Nrf2 gene and protein expression. Our experiments only examined 
the effects of endothelial damage in HMVEC cells, and we need a vivo experiment of carnosol to improve the diabetic microangiopathy. In addition, we can conduct an intensive study of the potential mechanisms in the future.

\section{Materials and Methods}

\subsection{Chemicals and Reagents}

Carnosol was purchased from Solarbio (Beijing, China). Dulbecco's modified eagle medium (DMEM) was purchased from Gibco (Grand Island, NY, USA). Fetal bovine serum (FBS) were purchased from Gibco BRL (Grand Island, NY, USA). Tert-Butyl hydroperoxide solution (t-BHP) was purchased from Macklin (Shanghai, China). PBS was obtained from Solarbio (PH 7.2-7.4) (Beijing, China). DCFH-DA was purchased from MedChemExpress (Monmouth Junction, NJ, USA).

\subsection{Cell Line and Culture}

Human lung microvascular endothelial cells (HMVEC-L) and Human Embryonic Kidney Cells (HEK293) cell line was purchased from ATCC. Cells were maintained in DMEM supplemented with $10 \%$ FBS and $0.29 \mathrm{~g} / \mathrm{L}$ L-glutamine, penicillin/streptomycin (1:100), in a humidified incubator at $37^{\circ} \mathrm{C}$ and $5 \% \mathrm{CO}_{2}$.

\subsection{Antioxidant Activity by ABTS Method}

Carnosol was dissolved in DMSO at a concentration of $40 \mathrm{mM}$ for stock solution. Antioxidant activity detected by ABTS kit (Beyotime, Shanghai, China). The ABTS solution mixed with $\mathrm{K}_{2} \mathrm{~S}_{2} \mathrm{O}_{8}$ to form a work solution and was stored at room temperature for $12 \mathrm{~h}-16 \mathrm{~h}$ before being used. Then the ABTS work solution was diluted with PBS for 40 times. $20 \mu \mathrm{L}$ of carnosol at various concentration $(10 \sim 0.04 \mu \mathrm{M})$ was mixed with $180 \mu \mathrm{L}$ of diluted ABTS work solution. The absorbance at $734 \mathrm{~nm}$ was measured after incubation for 2-6 $\mathrm{min}$ at room temperature. We use the Absorbance (ODs) function of Flex station 3 (Molecular Devices, San Francisco, CA, USA) to detect the absorbance.

\subsection{CCK-8 Assay for Cell Viability Evaluation}

HMVEC cells at a density of 9,000 per well were seeded in 96-well plates, incubated at $37^{\circ} \mathrm{C}$ for $24 \mathrm{~h}$, and treated with concentrations of carnosol for the $24 \mathrm{~h}$, the cytotoxicity of carnosol was first assessed by the Cell Counting Kit-8 (CCK-8) assay (MCE, Monmouth Junction, NJ, USA). The cells were incubated with CCK-8 reagent $(100 \mu \mathrm{L} / \mathrm{mL}$ medium) for $2 \mathrm{~h}$, and the absorbance was read at $450 \mathrm{~nm}$. Then they were treated with $200 \mu \mathrm{M}$ t-BHP for $3 \mathrm{~h}$. At the end of experiment, cell viability was evaluated by CCK-8 kit.

\subsection{Determination of $L D H$}

HMVEC were seeded in 96-well plates, incubated at $37^{\circ} \mathrm{C}$ for $24 \mathrm{~h}$, treated with carnosol for $24 \mathrm{~h}$ and then were treated with $200 \mu \mathrm{M} \mathrm{t}$-BHP for $3 \mathrm{~h}$. At the end of experiment, the supernatant for detecting the LDH release rate was sucked up with the LDH kits (Beyotime, Shanghai, China). This is a diaphorase-based INT color reaction. The LDH work solution was prepared according to the manufacturer's protocols. The next steps involved pipetting $120 \mu \mathrm{L}$ supernatant of each well which was added to the corresponding well of a new 96-well plate, then $60 \mu \mathrm{L} \mathrm{LDH}$ detection work solution was added to each well. The absorbance was read at $490 \mathrm{~nm}$.

\subsection{Annexin V-FITC/PI Double Staining}

HMVEC cells were seeded in 96-well plates, incubated at $37^{\circ} \mathrm{C}$ for $24 \mathrm{~h}$, and pre-incubated carnosol for the $24 \mathrm{~h}$ and then were treated with $200 \mu \mathrm{M}$ t-BHP for $3 \mathrm{~h}$. After being washed with PBS, $100 \mu \mathrm{L} 1 \times$ binding buffer was added, and was stained with $5 \mu \mathrm{L}$ Annexin-V and $5 \mu \mathrm{L}$ PI, then a fluorescence microscope was used to take photos. HMVEC cells at a density of 300,000 per well were 
seeded in 6-well plates, then treated with the same treatment for detection with a FACSC alibur flow cytometer (BD Sciences, San Jose, CA, USA).

\subsection{Immunofluorescence}

HMVEC cells $\left(1.5 \times 10^{4}\right.$ cells/well) were seeded in a 96-well plate and were treated with $10 \mu \mathrm{M}$ carnosol for $24 \mathrm{~h}$. Then, the cells were treated with $200 \mu \mathrm{M} \mathrm{t}$-BHP for $3 \mathrm{~h}$ and incubated with primary rabbit anti-VE Cadherin antibody (Abcam, Cambridge, UK) at $4{ }^{\circ} \mathrm{C}$ overnight. The cells were incubated with IgG H\&L (Alexa Flour ${ }^{\circledR} 488$ ) for $1 \mathrm{~h}$, and nuclei were covered with hoechest for $10 \mathrm{~min}$. The fluorescence signals were imaged using fluorescence microscope (LEICA, Solms, Germany). We analyzed the fluorescence intensity of the line in Merged pictures via Image-Pro Plus.

\subsection{Measurement of ROS and NO Generation}

HMVEC cells were seeded in 6-well plates and treated with carnosol for the $24 \mathrm{~h}$, then they were treated with $200 \mu \mathrm{M}$ t-BHP for $3 \mathrm{~h}$. The intracellular accumulation of ROS was detected using the DCFH-DA fluorescence assay. ROS generation was measured by the fluorescence intensity of dichlorofluorescein (DCF) at $525 \mathrm{~nm}$ after excitation at $488 \mathrm{~nm}$ using BD FACSC alibur flow cytometer. NO was detected by NO kit (Beyotime, Shanghai, China). The cells were loaded with DAF-FM DA (final concentration, $5 \mu \mathrm{M}$ ) for detecting the NO. Then we used a fluorescence microscope to take photos and analyzed the photos via Image J.

\subsection{Molecular Docking Analysis}

The crystal structure in complex with an inhibitor of Keap1 named 1VV (PDB code 4L7B, resolution $2.41 \AA$ ) was downloaded from the Protein Data Bank (www.rcsb.org). Excess protein side chain, incomplete residues, lack of hydrogen and the existence of waters molecules, were cleaned up by Discovery Studio 4.0 Client (DS 4.0). The active pocket was defined around 1VV. Then, carnosol was docked into the active pockets using CDOCKER methods.

\subsection{Luciferase Reporter Activity Assay}

To determine the ARE-agonistic activity of carnosol, we established a HEK293-ARE cell line. Luciferase Reporter Gene Assay Kit (Beyotime, Shanghai, China) was used to determine reporter gene activity in transiently transfected cells with FuGENE ${ }^{\circledR} 6$ Transfection Reagent (Promega, Madison, WI, USA). After transfection, cells at a density of $2.5 \times 10^{4}$ per well were seeded in 96 -well plates, incubated at $37^{\circ} \mathrm{C}$ for $24 \mathrm{~h}$, were treated with t-BHQ and carnosol (positive control) for $24 \mathrm{~h}$. Cell lysates were then prepared for the assessment of luciferase activity.

\subsection{1. $m R N A$ Quantification by Real-Time PCR}

HMVEC cells $\left(3 \times 10^{5}\right.$ cells/well) were seeded in a 6-well plate and were treated with carnosol for $24 \mathrm{~h}$ and then were treated with $200 \mu \mathrm{M}$ t-BHP for $3 \mathrm{~h}$. Total RNA was isolated from HMVEC cells using TRIzol Reagent (Life Technologies, Carlsbad, CA, USA) according to the manufacturer's protocol. After extracting total RNA from HMVEC cells, reverse transcription was performed prior to SYBR green real-time PCR analysis. Detection system is MA-6000 (Suzhou, China). The primers of objective genes and GAPDH primers were designed and shown in Table 1. 
Table 1. Primer sequences used for qRT-PCR analysis.

\begin{tabular}{|c|c|c|}
\hline Gene & Sequence $\left(5^{\prime}-3^{\prime}\right)$ & Product Length $(\mathrm{bp})$ \\
\hline HO-1 F & ACTGCGTTCCTGCTCAACAT & \multirow{2}{*}{133} \\
\hline$H O-1 R$ & GGGCAGAATCTTGCACTTTGT & \\
\hline$N r f 2 F$ & TCTGCCAACTACTCCCAGGT & \multirow{2}{*}{124} \\
\hline$N r f 2 R$ & ACGTAGCCGAAGAAACCTCA & \\
\hline eNOS F & GCCGGAACAGCACAAGAGTTA & \multirow{2}{*}{147} \\
\hline eNOS R & GCCCGAACACACAGAACCT & \\
\hline GAPDH F & CTTTGTCAAGCTCATTTCCTGG & \multirow[b]{2}{*}{133} \\
\hline GAPDH R & TCTTCCTCTTGTGCTCTTGC & \\
\hline
\end{tabular}

\subsection{Assessment Expression of HO-1 and Nrf2 Proteins}

Antibodies for HO-1, $\beta$-actin were purchased from Proteintech (Rosemont, IL, USA), antibody for Nrf2 was purchased from Abcam (Cambridge, UK). HMVEC cells were seeded in a 6-well plate and were treated with carnosol for $24 \mathrm{~h}$ with different doses of carnosol or for the indicated time. The protein bands were detected by ECL reagents with the Bio-Rad ChemiDocTM MP Imaging System (Hercules, CA, USA).

\subsection{Statistical Analyses}

Data is expressed as mean with standard deviation (SD). Analysis of variance (ANOVA) using Tukey's test was applied to compare the mean of each group with that of the control group, and $p$ value $<0.05$ was considered to be statistically significant.

Author Contributions: Y.Q. and Y.Z. (Yanling Zhang) conceived designed the project. X.L. designed and performed most experiments and wrote the paper. Q.Z. performed the luciferase reporter activity experiments. N.H., J.L. and M.L. helped to prepare the experiments. S.P., Y.Z. (Yuxin Zhang) and S.W. helped to prepare the compounds. Y.L., B.Z. helped to Building molecular docking model. All authors revised and approved the manuscript for publication.

Funding: This work supported by a grant from the National Natural Science Foundation of China (No. 81430094; No. 81573831), Beijing University of Chinese Medicine Key Laboratory Project and Beijing Education Committee Co-construction Project.

Conflicts of Interest: The authors declare no conflict of interest.

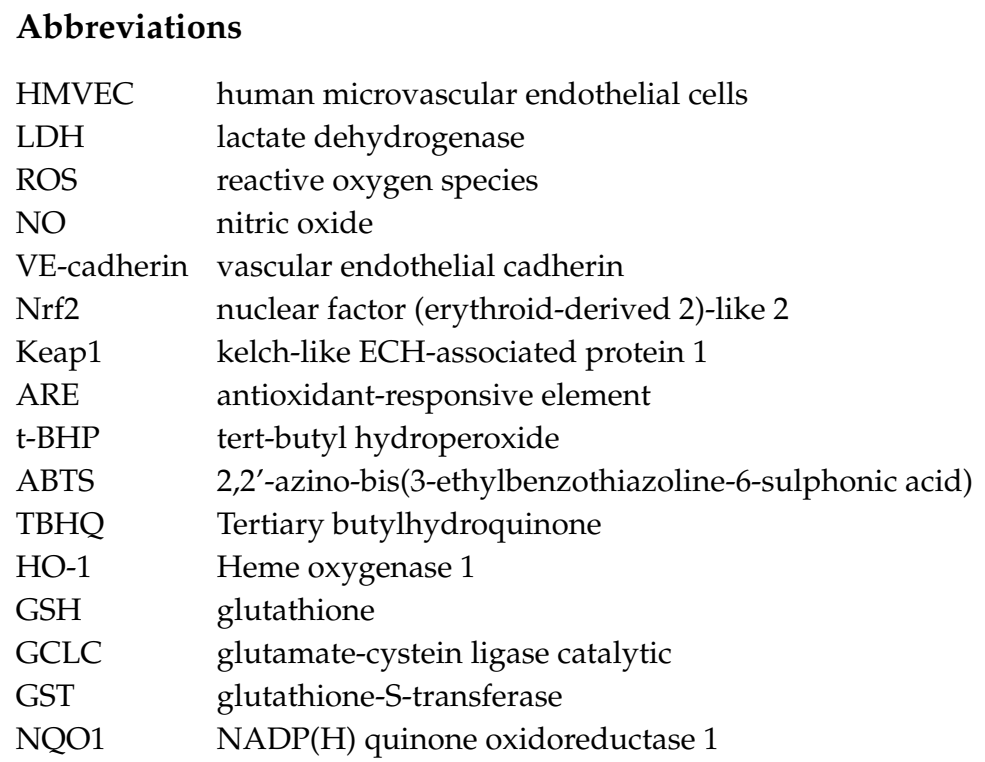




\section{References}

1. Patel, H.; Chen, J.; Das, K.C.; Kavdia, M. Hyperglycemia induces differential change in oxidative stress at gene expression and functional levels in huvec and hmvec. Cardiovasc. Diabetol. 2013, 12, 142. [CrossRef] [PubMed]

2. Wu, Z.; Wang, Z.; Dai, F.; Liu, H.; Ren, W.; Chang, J.; Li, B.; Wu, Z.; Road, J. Dephosphorylation of y685-ve-cadherin involved in pulmonary microvascular endothelial barrier injury induced by angiotensin ii. Mediat. Inflamm. 2016, 2016, 1-10. [CrossRef] [PubMed]

3. Giannotta, M.; Trani, M.; Dejana, E. Ve-cadherin and endothelial adherens junctions: Active guardians of vascular integrity. Dev. Cell 2013, 26, 441-454. [CrossRef] [PubMed]

4. Zou, Y.; Kim, D.; Yagi, M.; Yamasaki, Y.; Kurita, J.; Iida, T.; Matsuyama, Y.; Yamaguchi, K.; Oda, T. Application of ldh-release assay to cellular-level evaluation of the toxic potential of harmful algal species. Biosci. Biotechnol. Biochem. 2013, 77, 345-352. [CrossRef] [PubMed]

5. Li, H.; Horke, S.; Forstermann, U. Vascular oxidative stress, nitric oxide and atherosclerosis. Atherosclerosis 2014, 237, 208-219. [CrossRef] [PubMed]

6. Brownlee, M. Biochemistry and molecular cell biology of diabetic complications. Nature 2001, 414, 813-820. [CrossRef]

7. Ramalingam, M.; Kim, S.J. Insulin on hydrogen peroxide-induced oxidative stress involves ros/ca2+ and akt/bcl-2 signaling pathways. Free Radic. Res. 2014, 48, 347-356. [CrossRef]

8. Wu, C.R.; Chang, H.C.; Cheng, Y.D.; Lan, W.C.; Yang, S.E.; Ching, H. Aqueous extract of davallia mariesii attenuates 6-hydroxydopamine-induced oxidative damage and apoptosis in b35 cells through inhibition of caspase cascade and activation of pi3k/akt/gsk-3beta pathway. Nutrients 2018, 10, 1449. [CrossRef]

9. Bo, C.; Lu, Y.; Chen, Y.; Cheng, J. The role of nrf2 in oxidative stress-induced endothelial injuries. J. Endocrinol. 2015, 225, 83-99.

10. Santos, A.L.; Sinha, S.; Lindner, A.B. The good, the bad, and the ugly of ros: New insights on aging and aging-related diseases from eukaryotic and prokaryotic model organisms. Oxidative Med. Cell. Longev. 2018, 2018, 1-23. [CrossRef]

11. Jakaria, M.; Cho, D.Y.; Haque, M.E.; Karthivashan, G.; Kim, I.S.; Ganesan, P.; Choi, D.K. Neuropharmacological potential and delivery prospects of thymoquinone for neurological disorders. Oxidative Med. Cell. Longev. 2018, 2018, 1-17. [CrossRef] [PubMed]

12. Uruno, A.; Yagishita, Y.; Yamamoto, M. The keap1-nrf2 system and diabetes mellitus. Arch. Biochem. Biophys. 2015, 566, 76-84. [CrossRef] [PubMed]

13. Xue, M.; Qian, Q.; Adaikalakoteswari, A.; Rabbani, N.; Babaei-Jadidi, R.; Thornalley, P.J. Activation of nf-e2-related factor-2 reverses biochemical dysfunction of endothelial cells induced by hyperglycemia linked to vascular disease. Diabetes 2008, 57, 2809-2817. [CrossRef] [PubMed]

14. Katsuoka, F.; Motohashi, H.; Ishii, T.; Aburatani, H.; Engel, J.D.; Yamamoto, M. Genetic evidence that small maf proteins are essential for the activation of antioxidant response element-dependent genes. Mol. Cell. Biol. 2005, 25, 8044. [CrossRef] [PubMed]

15. Hayes, J.D.; Mcmahon, M.; Chowdhry, S.; Dinkova-Kostova, A.T. Cancer chemoprevention mechanisms mediated through the keap1-nrf2 pathway. Antioxid. Redox Signal. 2010, 13, 1713-1748. [CrossRef] [PubMed]

16. Zhou, M.X.; Li, G.H.; Sun, B.; Xu, Y.W.; Li, A.L.; Li, Y.R.; Ren, D.M.; Wang, X.N.; Wen, X.S.; Lou, H.X. Identification of novel nrf2 activators from cinnamomum chartophyllum $\mathrm{h}$. W. Li and their potential application of preventing oxidative insults in human lung epithelial cells. Redox Biol. 2017, 14, 154. [CrossRef] [PubMed]

17. Raskovic, A.; Milanovic, I.; Pavlovic, N.; Cebovic, T.; Vukmirovic, S.; Mikov, M. Antioxidant activity of rosemary (rosmarinus officinalis 1.) essential oil and its hepatoprotective potential. BMC Complement. Altern. Med. 2014, 14, 225. [CrossRef] [PubMed]

18. Sanchez, C.; Horcajada, M.N.; Scalfo, F.M.; Ameye, L.; Offord, E.; Henrotin, Y. Carnosol inhibits pro-inflammatory and catabolic mediators of cartilage breakdown in human osteoarthritic chondrocytes and mediates cross-talk between subchondral bone osteoblasts and chondrocytes. Plos One 2015, 10, e0136118. [CrossRef] [PubMed]

19. Oliviero, F.; Scanu, A.; Zamudio-Cuevas, Y.; Punzi, L.; Spinella, P. Anti-inflammatory effects of polyphenols in arthritis. J. Sci. Food Agric. 2017, 98, doi. [CrossRef] [PubMed] 
20. Yao, H.; Chen, Y.; Zhang, L.; He, X.; He, X.; Lian, L.; Wu, X.; Lan, P. Carnosol inhibits cell adhesion molecules and chemokine expression by tumor necrosis factor- $\alpha$ in human umbilical vein endothelial cells through the nuclear factor-kb and mitogen-activated protein kinase pathways. Mol. Med. Rep. 2014, 9, 476-480. [CrossRef] [PubMed]

21. Lazaro, I.; Lopez-Sanz, L.; Bernal, S.; Oguiza, A.; Recio, C.; Melgar, A.; Jimenez-Castilla, L.; Egido, J.; Madrigal-Matute, J.; Gomez-Guerrero, C. Nrf2 activation provides atheroprotection in diabetic mice through concerted upregulation of antioxidant, anti-inflammatory, and autophagy mechanisms. Front. Pharmacol. 2018, 9, 819. [CrossRef] [PubMed]

22. Banerjee, J.; Das, A.; Sinha, M.; Saha, S. Biological efficacy of medicinal plant extracts in preventing oxidative damage. Oxid. Med. Cell. Longev. 2018, 2018, 7904349. [CrossRef] [PubMed]

23. Kashyap, D.; Kumar, G.; Sharma, A.; Sak, K.; Tuli, H.S.; Mukherjee, T.K. Mechanistic insight into carnosol-mediated pharmacological effects: Recent trends and advancements. Life Sci. 2017, 169, $27-36$. [CrossRef] [PubMed]

24. Akbari Fakhrabadi, M.; Zeinali Ghotrom, A.; Mozaffari-Khosravi, H.; Hadi Nodoushan, H.; Nadjarzadeh, A. Effect of coenzyme q10 on oxidative stress, glycemic control and inflammation in diabetic neuropathy: A double blind randomized clinical trial. Int. J. Vitam. Nutr. Res. 2014, 84, 252-260. [CrossRef] [PubMed]

25. Arora, M.K.; Singh, U.K. Oxidative stress: Meeting multiple targets in pathogenesis of diabetic nephropathy. Curr. Drug Targets 2014, 15, 531-538. [CrossRef] [PubMed]

26. Kowluru, R.A.; Kowluru, A.; Mishra, M.; Kumar, B. Oxidative stress and epigenetic modifications in the pathogenesis of diabetic retinopathy. Prog. Retin. Eye Res. 2015, 48, 40-61. [CrossRef]

27. Ewald, C.Y.; Hourihan, J.M.; Blackwell, T.K. Oxidative stress assays (arsenite and tbhp) in caenorhabditis elegans. Bio. Protoc. 2017, 7. [CrossRef] [PubMed]

28. Manu, T.M.; Anand, T.; Khanum, F. Attenuation of cytotoxicity induced by tbhp in h9c2 cells by bacopa monniera and bacoside a. Pathophysiology 2018, 25, 143-149.

29. Wang, D.; Chen, T.; Liu, F. Betulinic acid alleviates myocardial hypoxia/reoxygenation injury via inducing nrf2/ho-1 and inhibiting p38 and jnk pathways. Eur. J. Pharmacol. 2018, 838, 53-59. [CrossRef] [PubMed]

30. Zhang, S.L.; Du, Y.H.; Wang, J.; Yang, L.H.; Yang, X.L.; Zheng, R.H.; Wu, Y.; Wang, K.; Zhang, M.S.; Liu, H.R. Endothelial dysfunction induced by antibodies against angiotensin at1 receptor in immunized rats. Acta Pharmacol. Sin. 2010, 31, 1381-1388. [CrossRef] [PubMed]

31. Schor, N.F. Apoptosis in the Nervous System. In The Neurology of Neuroblastoma; Springer: Boston, MA, USA, 2002.

32. Yang, B.; Cai, B.; Deng, P.; Wu, X.; Guan, Y.; Zhang, B.; Cai, W.; Schaper, J.; Schaper, W. Nitric oxide increases arterial endotheial permeability through mediating ve-cadherin expression during arteriogenesis. PLoS ONE 2015, 10, e0127931. [CrossRef] [PubMed]

33. Murphy, M.P. Antioxidants as therapies: Can we improve on nature? Free Radic. Biol. Med. 2014, 66, $20-23$. [CrossRef] [PubMed]

34. Selimoglu-Buet, D.; Badaoui, B.; Benayoun, E.; Toma, A.; Fenaux, P.; Quesnel, B.; Etienne, G.; Braun, T.; Abermil, N.; Morabito, M.; et al. Accumulation of classical monocytes defines a subgroup of mds that frequently evolves into cmml. Blood 2017, 130, 832-835. [CrossRef] [PubMed]

35. Stefanson, A.L.; Marica, B. Dietary regulation of keap1/nrf2/are pathway: Focus on plant-derived compounds and trace minerals. Nutrients 2014, 6, 3777-3801. [CrossRef] [PubMed]

36. Long, M.; de la Vega, M.R.; Wen, Q.; Bharara, M.; Jiang, T.; Zhang, R.; Zhou, S.; Wong, P.K.; Wondrak, G.T.; Zheng, H.; et al. An essential role of nrf2 in diabetic wound healing. Diabetes 2016, 65, 780-793. [CrossRef] [PubMed]

37. Cullinan, S.B.; Zhang, D.; Hannink, M.; Arvisais, E.; Kaufman, R.J.; Diehl, J.A. Nrf2 is a direct perk substrate and effector of perk-dependent cell survival. Mol. Cell Biol. 2003, 23, 7198-7209. [CrossRef] [PubMed]

38. Feng, X.E.; Wang, Q.J.; Gao, J.; Ban, S.R.; Li, Q.S. Synthesis of novel nitrogen-containing heterocycle bromophenols and their interaction with keap1 protein by molecular docking. Molecules 2017, 22, 2142. [CrossRef]

39. Jiang, Z.Y.; Chu, H.X.; Xi, M.Y.; Yang, T.T.; Jia, J.M.; Huang, J.J.; Guo, X.K.; Zhang, X.J.; You, Q.D.; Sun, H.P. Insight into the intermolecular recognition mechanism between keap1 and ikkbeta combining homology modelling, protein-protein docking, molecular dynamics simulations and virtual alanine mutation. PLOS ONE 2013, 8, e75076. 
40. Amata, E.; Pittala, V.; Marrazzo, A.; Parenti, C.; Prezzavento, O.; Arena, E.; Nabavi, S.M.; Salerno, L. Role of the nrf2/ho-1 axis in bronchopulmonary dysplasia and hyperoxic lung injuries. Clin. Sci. 2017, 131, 1701-1712. [CrossRef]

41. Luo, Y.; Lu, S.; Dong, X.; Xu, L.; Sun, G.; Sun, X. Dihydromyricetin protects human umbilical vein endothelial cells from injury through erk and akt mediated nrf2/ho-1 signaling pathway. Apoptosis 2017, 22, 1013-1024. [CrossRef]

(c) (C) 2019 by the authors. Licensee MDPI, Basel, Switzerland. This article is an open access article distributed under the terms and conditions of the Creative Commons Attribution (CC BY) license (http://creativecommons.org/licenses/by/4.0/). 